\title{
AROMI NATURALI: LEGISLAZIONE E SINTESI
}

\author{
STEFANO SERRA (*) \\ Nota presentata dal m.e. C. Fuganti \\ (Adunanza del 12 aprile 2018)
}

SunTO. - In questa nota sono descritte le principali normative che regolano la produzione, classificazione e commercializzazione degli aromi. Viene anche descritto in dettaglio l'effetto che la normativa stessa ha portato nell'ambito dello sviluppo di nuovi metodi di sintesi degli aromi naturali.

$* * *$

ABSTRACT. - This report describes the legal regulations concerning flavour production, classification and marketing. The effect that the legislation itself has brought in the development of new methods of synthesis of natural flavors is described in detail.

\section{INTRODUZIONE}

Con il termine aroma si intendono sostanze utilizzabili in alimenti. Le fragranze sono invece impiegate per uso esterno (profumi). La distinzione non è sempre netta, esistono sostanze aromatiche utilizzabili come fragranze (mentolo, linalolo).

Il mercato globale degli aromi e delle fragranze è in continua crescita. Nel 2015 ha raggiunto un valore complessivo di 23.6 miliardi di dollari, di cui circa il 55\% legato alla vendita di aromi. Stime di mercato prevedono un aumento di crescita globale corrispondente al $5 \%$ annuo

(*) C.N.R., Istituto di Chimica del Riconoscimento Molecolare (ICRM), Milano, Italy. E-mail: stefano.serra@cnr.it; stefano.serra@polimi.it 
almeno fino al 2020. I produttori di aromi sono essenzialmente le industrie chimiche. Una parte consistente degli aromi presenti sul mercato è di origine estrattiva ma l'industria chimica è produttrice sia di aromi 'naturali' sia di aromi 'sintetici'.

In questo contesto, i consumatori richiedono preparazioni che contengano aromi 'naturali'. Il termine naturale lascia intendere che la sostanza venga dal mondo vegetale, non sia prodotta industrialmente e non siano stati usati OGM o reagenti chimici per produrla. Sull'onda di queste preferenze sono nate quindi molte certificazioni quali 'aroma naturale', 'OGM free' e 'biologico' (organic), nonché certificazioni legate a specifiche regole religiose quali 'Kosher' o 'Halal'.

Come viene regolata da un punto di vista normativo la produzione ed il commercio di questi prodotti? Quale effetto hanno avuto le normative vigenti nell'ambito della chimica degli aromi?

La relazione che segue cercherà di rispondere a queste domande.

\section{CHIMICA E QUADRO NORMATIVO}

Da un punto di vista chimico non c'è nessuna differenza tra un dato composto chimico prodotto per sintesi e lo stesso composto estratto da una fonte naturale. A seconda del processo produttivo si possono avere differenze nell'ambito del profilo delle impurezze, della purezza isomerica (nel caso di stereoisomeri) o dell'abbondanza isotopica di specifici atomi presenti nella molecola ma i singoli composti chimici sono identici.

Il punto cardine della normativa vigente risiede nell'ammissibilità di un dato composto aromatico negli alimenti e nella definizione di naturale. I criteri di ammissibilità e naturalità possono differire da stato a stato. Le organizzazioni legate alla sicurezza alimentare indicano gli aromi ritenuti sicuri e chiedono l'eliminazione di quelli potenzialmente pericolosi. Contrariamente all'opinione comune molte sostanze naturali sono tossiche ed è possibile che esistano aromi 'naturali' non ammessi in alimenti.

L'industria chimica necessita di un quadro normativo chiaro. La produzione si adatta alle richieste di mercato a patto che la preparazione di 'aromi naturali' sia profittevole e sia possibile certificare l'origine (la naturalità). Di conseguenza diverse organizzazioni internazionali si sono occupate di creare direttive su questa tematica. Le più importanti 
sono elencate di seguito, con annessa la descrizione della loro principale attività (descrizione ottenuta dallo statuto dell'organizzazione stessa)

- CODEX ALIMENTARIUS: Currently the Codex Alimentarius Commission has 189 Codex Members made up of 188 Member Countries and 1 Member Organization (The European Union). The Codex Alimentarius is intended to guide and promote the elaboration and establishment of definitions and requirements for foods to assist in their harmonization and in doing so to facilitate international trade.

- JECFA: Joint FAO/WHO Expert Committee on Food Additives. JECFA is an international scientific expert committee administered jointly by the Food and Agriculture Organization of the United Nations (FAO) and WHO.

- FEMA: Flavor and Extract Manufacturers Association of the United States. FEMA is committed to assuring a substantial supply of safe flavoring substances.

- EFSA: European Food Safety Authority. L'Autorità europea per la sicurezza alimentare (EFSA) fornisce consulenze scientifiche indipendenti sui rischi connessi all'alimentazione.

- FCC: Food Chemicals Codex is a collection of internationally recognized standards for the purity and identity of food ingredients.

Sebbene le organizzazioni internazionali abbiano fornito importanti line guida (ad esempio CODEX ALIMENTARIUS ha fornito la Codex Guidelines for Flavoring CAC-GL 66/2008), la produzione mondiale degli aromi naturali è influenzata principalmente dall'attività legislative di Stati Uniti ed Unione Europea in cui si colloca una grossa fetta del mercato mondiale degli aromi naturali.

Altri stati quali Cina, Giappone, India, Federazione Russa, e paesi del Mercosur (Mercato comune dell'America meridionale) sebbene siano grossi produttori e consumatori di aromi naturali hanno una legislazione meno rigida rispetto a Stati Uniti ed Unione Europea.

In passato gli aromi venivano divisi in tre classi (Codex Food Additives and Contaminants (CCFAC) - 1972 / Codex Food Labelling (CCFL) - 1985):

- Aromi naturali (estratti da fonti naturali o preparati da precursori naturali con metodi naturali).

- Aromi naturali identici (aromi prodotti per sintesi ma chimicamente identici ad aromi naturali).

- Aromi Artificiali (aromi prodotti per sintesi, non presenti in natura). 
Questa classificazione è ancora valida in alcuni paesi quali ad esempio India e Brasile.

La classificazione CODEX in vigore identifica invece solo due tipi di aromi: aromi naturali e sostanze aromatiche sintetiche. Di seguito le definizioni (Codex Guidelines for Flavoring CAC-GL 66/2008):

Natural flavouring substances are flavouring substances obtained by physical processes that may result in unavoidable but unintentional changes in the chemical structure of the components of the flavouring (e.g. distillation and solvent extraction), or by enzymatic or microbiological processes, from material of plant or animal origin. Such material may be unprocessed, or processed for human consumption by traditional food-preparation processes (e.g. drying, torrefaction (roasting) and fermentation). This means substances that have been identified /detected in a natural material of animal or vegetable origin.

Synthetic flavouring substances are flavouring substances formed by chemical synthesis.

Anche l'Unione Europea identifica solo due categorie di aromi: aromi naturali ed aromi. La normativa di riferimento è il Regolamento $\mathbf{N}$. 1334/2008 del 16/12/2008 [1] ed in particolare l'art. 3, comma c recita:

\footnotetext{
' per «sostanza aromatizzante naturale» s'intende una sostanza aromatizzante ottenuta mediante appropriati procedimenti fisici, enzimatici o microbiologici da un materiale di origine vegetale, animale o microbiologica, che si trova allo stato grezzo o che è stato trasformato per il consumo umano mediante uno o più procedimenti tradizionali di preparazione degli alimenti di cui all'allegato II. Le sostanze aromatizzanti naturali corrispondono a sostanze normalmente presenti e identificate in natura;'
}

\section{AROMI E AROMI ARTIFICIALI, DUE MERCATI SEPARATI}

Sebbene con differenti sfumature, le normative vigenti contribuiscono a creare due mercati differenti. In particolare le regole stringenti delineate dai legislatori per regolamentare la produzione di aromi naturali rendono questi composti assimilabili a prodotti ad alto valore aggiunto, in quanto sono necessari processi molto più complessi e/o costosi per la loro preparazione. In particolare l'esigenza di sviluppare processi basati sull'uso di reazioni enzimatiche o trasformazioni microbiologiche ha favorito lo sviluppo di molti studi scientifici legati alle 
biotrasformazioni [2]. Sono illustrati di seguito due esempi rilevanti legati alla produzione industriale della vanillina e dei lattoni.

\subsection{Sintesi della vanillina}

La vanillina è un aroma di largo consumo. La vanillina preparata per sintesi chimica è molto meno costosa di quella naturale (estratta dalle bacche di vaniglia oppure ottenuta per trasformazione microbiologica). Nello schema seguente (Fig. 1) è descritto il principale metodo di sintesi della vanillina artificiale. I composti di partenza sono il guaiacolo e l'acido gliossilico, disponibili a basso prezzo in quanto preparati dall'industria petrolchimica. La sequenza di reazioni che viene usata prevede l'uso di reagenti molto semplici quali la soda caustica, l'ossigeno dell'aria ed una catalisi acida. Complessivamente il processo illustrato porta sul mercato vanillina ad alta purezza con un prezzo di circa 15 20 euro/Kg.

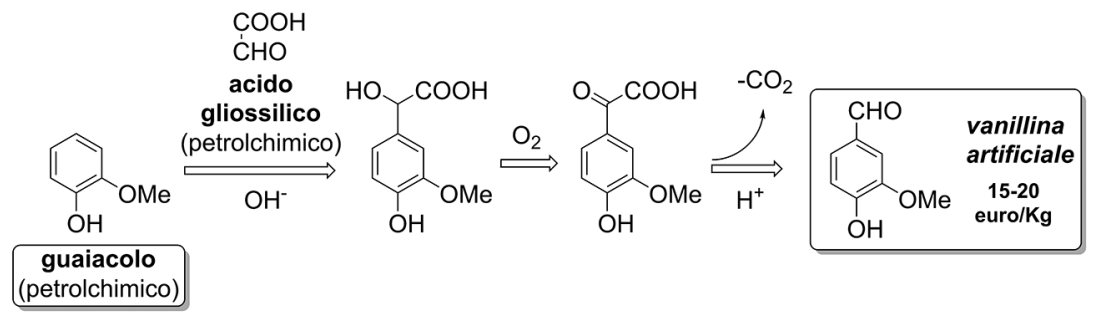

Fig. 1. Sintesi industriale della vanillina dal guaiacolo.

Del tutto diversi sono i processi per la preparazione della vanillina naturale (Fig. 2). Ne esistono molti e per poter essere conformi alla regolamentazione internazionale sono basati sull'utilizzo di precursori naturali (usualmente estrattivi) seguiti dalla loro biotrasformazione in vanillina [3]. L'estrazione della vanillina stessa dalle bacche è ancora un processo molto usato anche se tra i più costosi in termini di resa. Uno tra i processi biotecnologici più usati è basato sulla degradazione microbica dell'acido ferulico che viene a sua volta ottenuto per estrazione della pula di riso.

Altri processi sono basati sulla biotrasformazione di prodotti naturali quali l'eugenolo, l'isoeugenolo e l'isorapontina. Data la variabilità dei metodi di sintesi, il prezzo della vanillina naturale può passare dai 1000 euro/Kg ai 5000 euro/Kg (vanillina da bacche), quindi almeno cinquanta volte superiore a quello della vanillina artificiale. 


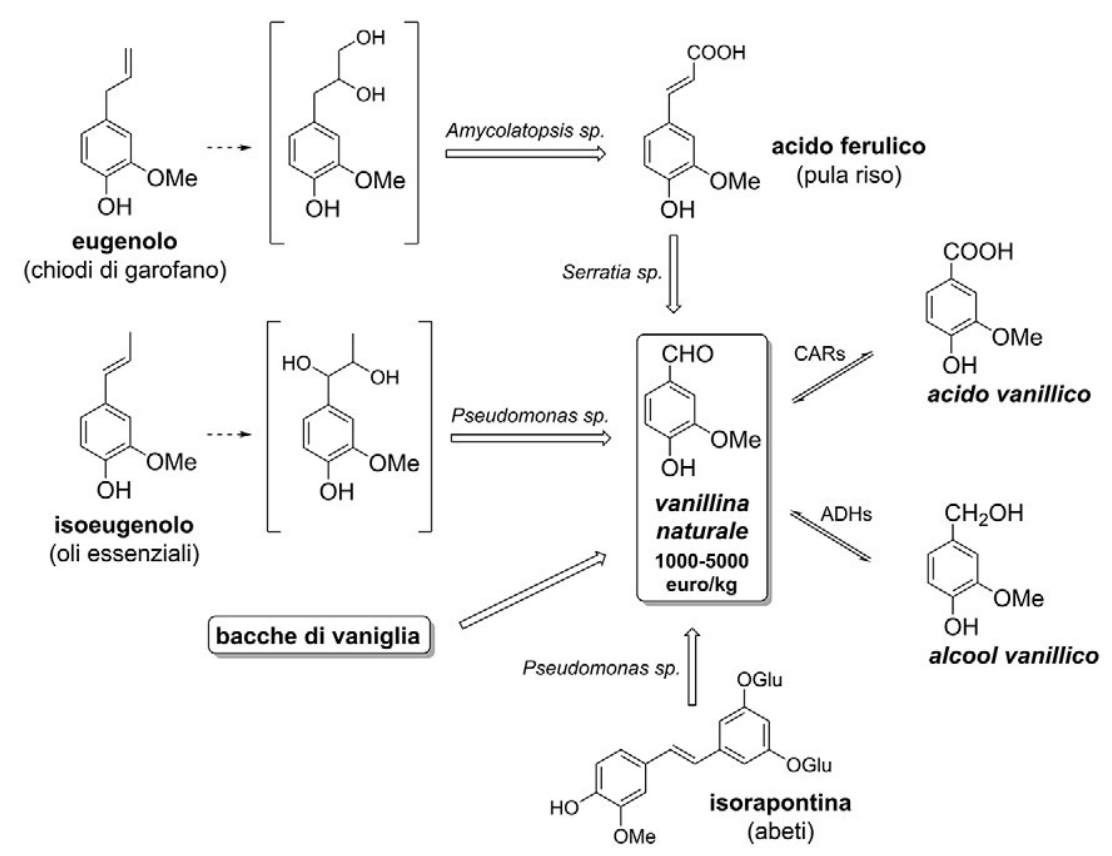

Fig. 2. Sintesi della vanillina naturale.

\subsection{Sintesi dei lattoni naturali}

I gamma-lattoni decalattone e dodecalattone sono composti largamente usati per conferire aroma pesca ad alimenti e bevande. Questi lattoni sono facilmente ottenibili per sintesi e sono quindi disponibili a basso prezzo. In natura sono però presenti solo in tracce e quindi l'estrazione dai vegetali che li contengono non è un processo fattibile. In questo ambito le biotecnologie hanno permesso la produzione di numerosi lattoni in forma naturale. Il decalattone (Fig. 3) è attualmente prodotto dall'olio di ricino mediante un processo a due steps [4]. Nel primo si idrolizza l'olio con una lipasi a dare l'acido ricinoleico. Successivamente l'acido viene degradato mediante una trasformazione microbica mediata da un lievito a dare il decalattone. Sebbene le rese complessive non siano elevate, l'olio di ricino ha un prezzo di pochi euro/Kg mentre il gamma-decalattone naturale è venduto a diverse centinaia di euro $/ \mathrm{Kg}$. IL processo è quindi particolarmente profittevole ed ha portato sul mercato una consistente quantità di questo lattone in forma naturale. 

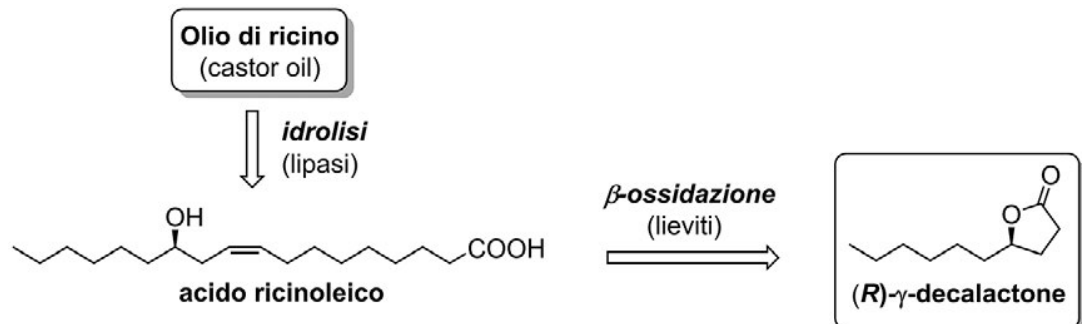

Fig. 3. Produzione industriale del gamma-decalattone naturale.

\section{CONCLUSIONI}

Le preferenze dei consumatori hanno influenzato marcatamente l'industria di produzione degli aromi. In particolare si sono creati due differenti mercati: quello degli aromi e quello degli aromi naturali. L'elevato valore aggiunto generato dalla classificazione 'naturale', ha spinto verso la creazione di nuove aziende biotecnologiche nonché lo studio di nuove procedure sintetiche che siano in accordo con la regolamentazione vigente nel campo degli aromi naturali. La necessità di tracciare/appurare la naturalità di un dato aroma, ha stimolato la ricerca nel campo analitico in particolare nell'ambito dell'autenticazione ed in quello dell'identificazione e caratterizzazione di nuovi aromi naturali.

\section{BIBLIOGRAFIA}

[1] Regolamento (CE) n. 1334/2008 del Parlamento europeo e del Consiglio, del 16 dicembre 2008 , relativo agli aromi e ad alcuni ingredienti alimentari con proprietà aromatizzanti destinati a essere utilizzati negli e sugli alimenti e che modifica il regolamento (CEE) n. 1601/91 del Consiglio, i regolamenti (CE) n. 2232/96 e (CE) n. 110/2008 e la direttiva 2000/13/CE (Testo rilevante ai fini del SEE).

[2] Serra, S.; Fuganti, C.; Brenna, E. Biocatalytic Preparation of Natural Flavours and Fragrances. Trends Biotechnol. 2005, 23 (4), 193-198. https://doi.org/ 10.1016/j.tibtech.2005.02.003.

[3] Kaur, B.; Chakraborty, D. Biotechnological and molecular approaches for vanillin production: A review. Appl. Biochem. Biotechnol. 2013, 169, 1353-1372.

[4] Wache, Y.; Aguedo, M.; Nicaud, J.-M.; Belin, J.-M. Catabolism of Hydroxyacids and Biotechnological Production of Lactones by Yarrowia Lipolytica. Appl. Microbiol. Biotechnol. 2003, 61 (5-6), 393-404. https://doi.org/10.1007/s00253002-1207-1. 
